\title{
Trends in the evolution of particle morphology with size in colluvial deposits overlying channel iron deposits
}

\author{
Sandra Linero ${ }^{1,2^{*}}$, Stephen Fityus ${ }^{1}$, John Simmons $^{3}$, Arcesio Lizcano ${ }^{4}$ and Jessica Cassidy ${ }^{1}$ \\ ${ }^{1}$ University of Newcastle, Faculty of Engineering and Build Environment, University Dr Callaghan NSW2308, Australia \\ ${ }^{2}$ SRK Consulting (Australasia) Pty Ltd, 10 Richardson St WA6005, Australia \\ ${ }^{3}$ Sherwood Geotechnical and Research Services, 5/8 Grebe St Peregian Beach QLD4573, Australia \\ ${ }^{4}$ SRK Consulting (Canada) Inc, 1066 West Hastings St, BC V6E 3X2, Canada
}

\begin{abstract}
Size limitations of testing equipment often impliy that samples of coarse granular materials must be scalped or scaled, to reduce the size of the constitutive particles, before they can be tested either by triaxial or direct shear in the laboratory. The objective of the investigation is to evaluate the particle shapes in a natural sample of colluvial sediments, to identify potential correlation(s) between shape and size, that could impact shear strength of scaled samples. The material investigated is derived from eroded ancient sedimentary rocks from the Pilbara region of Australia. The fragments have a particle shape ranging from slabs to sub-equant blocks. The observation indicates that there is an increase in the tendency for slabshapes in larger particles. Therefore, scaling inevitably alters the characteristic shapes of the material particles as it implies substituting larger (slabs) particles by smaller (sub-equant) particles. Changes in particle shape distribution may induce changes in material fabric and shear strength and therefore may need to be considered when scaling samples.
\end{abstract}

\section{Introduction}

The mechanical behaviour of granular materials is affected by the shape of the constituent particles. Experimental work suggests that the shear strength increases with particle angularity [1][2]. Micromechanical investigations using DEM suggest that the shear strength decreases with increasing shape polydispersity [3].

The objective of this investigation is to evaluate the particle shapes in a natural sample of colluvial sediments, to identify potential correlations between shape and size.

When a sample of granular material has very large particles, compared with the dimensions of the available shear testing device, manipulation of the particle size distribution (PSD) is required to obtain a maximum particle size $\left(\mathrm{d}_{\max }\right)$ that suits the available equipment. Parallel scaling is a common technique to reduce the maximum particle size of a granular sample (Figure 1).

Any of the common techniques for material scaling (parallel scaling or scalping) change the original particle size distribution and, potentially, the particle shape distribution. This is significant, however, because changes in particle shape distribution may conduce to a change in material fabric and shear strength. Therefore, potential correlation between particle- shape and size in natural granular materials may need to be considered when scaling samples for laboratory testing.

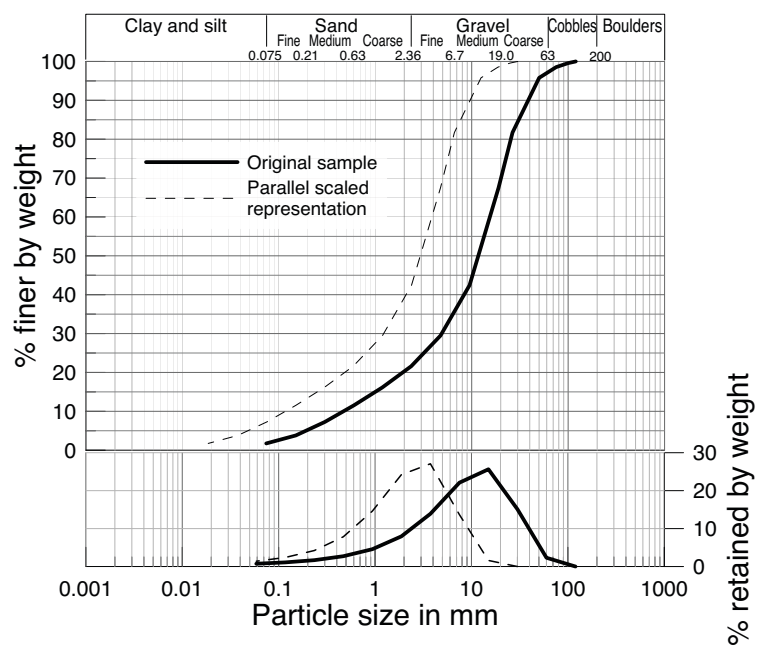

Fig. 1. PSD for the investigated sample $\left(\mathrm{d}_{\max } 120 \mathrm{~mm}\right)$ and its scaled representation $\left(\mathrm{d}_{\max } 30 \mathrm{~mm}\right)$ : Cumulative function (above), and variation of weight percentage as a continuum function of grain size (below)

\section{The sample}

The sample is an overburden material composed of eroded and transported particles (colluvium) derived from a sedimentary Precambrian-aged Banded Iron Formation (BIF), with province-wide distribution, located at the Pilbara region of Australia [4]. The BIF formations consist essentially of alternating beds and 
laminae of hematite or magnetite and chert. The sample material occurs in what are known as channel iron deposits, one of the three major styles of iron ore mineralisation that are developed and commercially exploited in the Hamersley Province [5]. Locally, the colluvium derived from the BIF outcrops forms densityseparated deposits in incised channels, with denser ironrich fragments (the orebody) in the bottom of the channel, overlain by chert-rich fragments (overburden).

Thin layering of banding of less than $1 \mathrm{~mm}$ to few centimetres can be observed in saw cut and polished fragments (Figure 2). Parting occurs preferentially along laminations. Parting along fractures sub-perpendicular to the lamination can occasionally be recognized. The evaluation of the relationship between particle size and lamination composition was not part of this research.

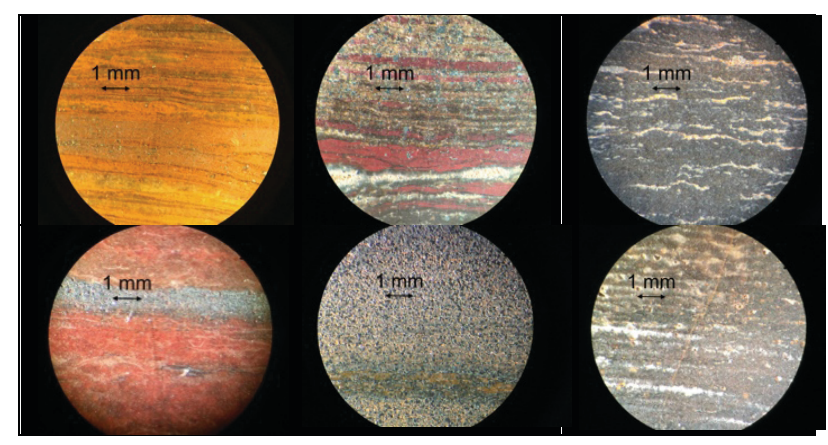

Fig. 2. 30X magnification of polished fragments (grey grains are hematite and magnetite; red and orange material is chert.)

A sample of $16.8 \mathrm{~kg}$ was obtained by dividing 1.4 tonnes of material using a rotary divider. The particle size distribution (PSD) was determined by dry sieving, using a standard set of meshes with opening sizes between $75 \mathrm{~mm}$ and $0.075 \mathrm{~mm}$.

Twelve different size fractions (Table 1) covering $95 \%$ of the mass of the material were defined for analysis.

Table 1. Weight and number of particles retained by sieve and number of particles selected for shape determination.

\begin{tabular}{crrcrc}
\hline No & $\begin{array}{r}\text { Sieve } \\
\text { Size } \\
(\mathrm{mm})\end{array}$ & \multicolumn{2}{c}{ Retained mass } & \multicolumn{2}{c}{ No of particles } \\
& $(\mathrm{gr})$ & $(\%)$ & \multicolumn{1}{c}{ Retained } & Analysed \\
\hline & 75 & 231 & 1.37 & $1^{(1)}$ & \\
1 & 50 & 199 & 1.18 & $1^{(1)}$ & $24^{(*)}$ \\
2 & 26.5 & 2346 & 13.89 & $44^{(1)}$ & 40 \\
3 & 19.0 & 2388 & 14.14 & $128^{(1)}$ & 117 \\
4 & 13.2 & 2556 & 15.14 & $372^{(1)}$ & 43 \\
5 & 9.5 & 1763 & 10.44 & $671^{(1)}$ & 161 \\
6 & 6.5 & 1230 & 7.28 & $1,230^{(2)}$ & 51 \\
7 & 4.75 & 980 & 5.80 & $2,571^{(2)}$ & 70 \\
8 & 2.36 & 1344 & 7.96 & $16,850^{(3)}$ & 75 \\
9 & 1.18 & 1020 & 6.04 & $51,298^{(3)}$ & 20 \\
10 & 0.6 & 789 & 4.67 & $531,388^{(3)}$ & 20 \\
11 & 0.3 & 731 & 4.33 & $2,334,654^{(3)}$ & 20 \\
12 & 0.15 & 617 & 3.66 & $16,621,585^{(3)}$ & 20 \\
& 0.075 & 387 & 2.29 & - & - \\
& Resid. & 305 & 1.81 & - & - \\
\hline
\end{tabular}

(*) Population augmented to improve observations
The numbers in parentheses in the Table 1 indicate how the number of particles was determined: (1) counting, (2) extrapolating the number of particles counted in $200 \mathrm{~g}$ of material, (3) dividing retained weight by average particle weight. The average particle weight was calculated from averaged volume and density of particles. The average volume was determined based on the average dimensions of the particles using a correction factor. The latter was backcalculated from a sub-sample of counted particles assuming similarity of large and small sizes.

Roundness determination was conducted on ten particles of each size fraction.

\section{Measurement protocols}

The shape of the particles was evaluated based on "elongation" and "flatness", considering the standard measurement protocol proposed by Krumbein [6]. Those descriptors are defined as the elongation (breadth to length) ratio $(I / L)$ and flatness (thickness to breadth) ratio $(S / I)$, respectively, where $L$ is the greatest distance apart of two parallel planes tangential to the particle surface, $I$ is the maximum dimension perpendicular to $L$, and $S$ is the maximum dimension perpendicular to $L$ and I. $L, I$ and $S$ define an imaginary box that contains the particle, as shown in Figure 3. This information allows classification of the shape of the particles in 8 different categories (illustrated in Figure 5) [7].

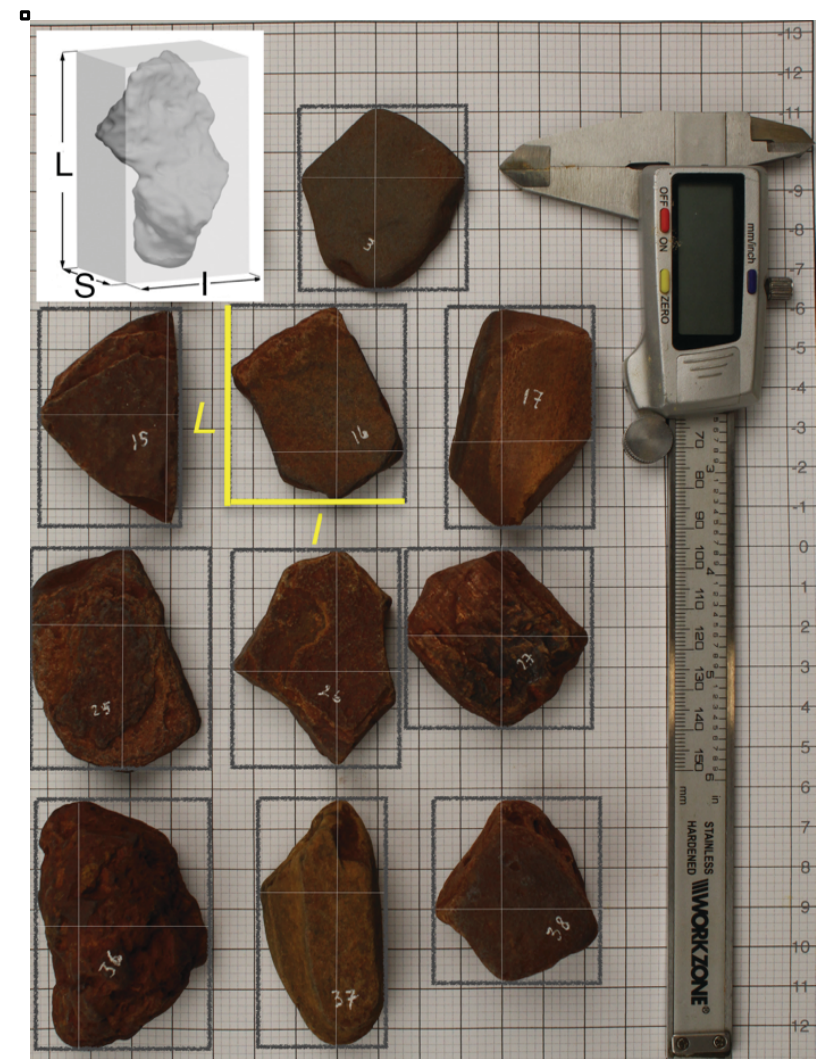

Fig. 3. Characteristic dimensions of the particles, $L=$ Length, $I=$ Breath and $S=$ Thickness. Insert imaged modified from [8].

The Krumbein protocol is well suited for rather ellipsoidal particles. If the distances $\mathrm{L}, \mathrm{I}$, and $\mathrm{S}$, are 
instead defined as those of the smallest rectangular box that contains the particle, then elongation and flatness ratio may vary especially for rather square parallelepiped pieces.

A calliper was used to determine dimensions of particles larger than $2.36 \mathrm{~mm}$. Each was placed on graph paper exposing the greatest area projection. By orienting the length of the particle with one of the lines of the grid, length $(L)$ and breadth $(I)$ orthogonality was ensured.

Smaller particles were placed under a microscope and photographed. The length and breadth of those small particles were determined by analysing the microscope images. Their thickness was defined using the microscope's depth measuring device.

The roundness was determined using the roundness coefficient $R r$ by Wadell [9] which evaluates the relative sharpness of particle corners. A corner is that part of the outline of the projection area which has a radius of curvature equal to or less than the radius of curvature of the maximum inscribed circle of the same projection area (Figure 4). The roundness coefficient is defined as the average radii of the corners divided by the radii of the inscribed circle, on the largest projected area of the particle. The coefficient allows classification into four categories: angular $0<R r<0.13$; sub-angular $0.13<R r<$ 0.25 ; sub-rounded $0.25<R r<0.5$ and rounded $0.5<R r<1$.

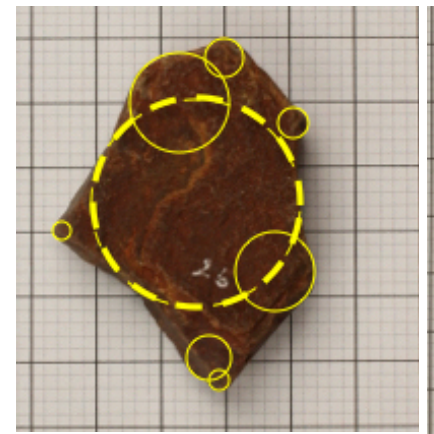

Sub-angul. $R r=0.17$

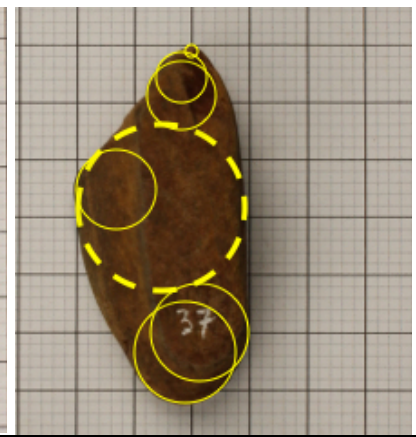

Sub-rounded $R r=0.33$
Fig. 4. Roundness determination showing maximum inscribed circle (thick dashed line) and corner circles (thin solid line).

\section{Results}

\subsection{Particle shape}

From the flatness vs. elongation data in Figure 5, most particles classify as slabs, and flat to sub equant blocks. The breadth to length ratios (elongation) are mostly greater than 0.5 , with a mean of around 0.75 , reflecting a tendency for the two largest dimensions to be relatively close in value. There is a much greater spread in flatness ratio compared with elongation ratio, with values relatively evenly distributed between 0.2 and 1.0, reflecting greater variability between the smallest dimension and the two larger dimensions. This outcome can be attributed to the sedimentary nature of the material, and the preferential weakness imparted by the laminations, resulting in a tendency for the minimum dimension being variable, and perpendicular to the laminations.

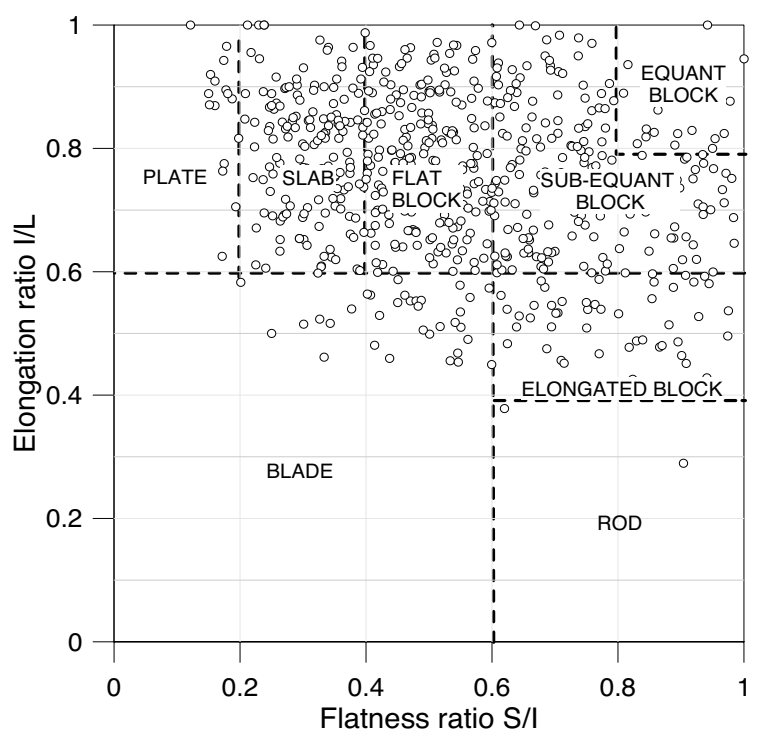

Fig. 5. Elongation vs Flatness for all the analysed particles.

The distribution of data by size fraction is illustrated in Figures 6,7 and 8. The notched boxes are drawn to represent the interquartile range of the data. The horizontal line inside indicates the median value. The lower and upper quartiles are shown as horizontal lines either side of the boxes. "Whiskers" above and below each box show the locations of the 5 and 95 percentile. The proportion of material mass by size fraction is also illustrated in the figures for the analysed sample and for its scaled representation, for comparison.

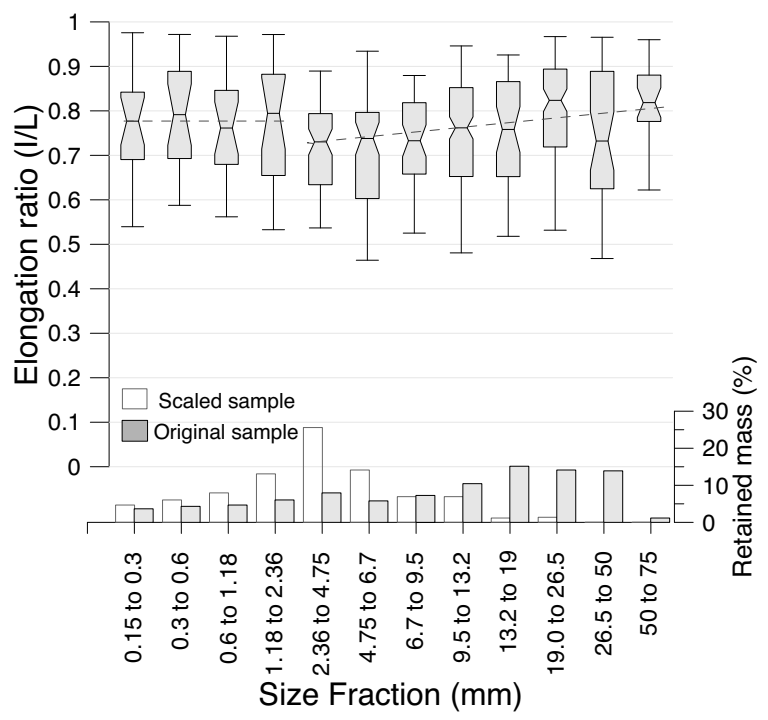

Fig. 6. Box plot showing the variability of elongation ratio, calculated from the form dimensions $L$ and $I$ for the different size fractions compared to the represented proportion of mass.

Trends of increasing elongation ratio and decreasing flatness ratio with size are observed for the particles between $2.36 \mathrm{~mm}$ and $75 \mathrm{~mm}$ (gravels and cobbles). In this range, larger particles tend to be less elongated and flatter than smaller particles (Figure 6 and 7). However, the elongation trend is not very significant, with median values varying less than $10 \%$ in the range. The variation in flatness ratio is more pronounced with up to $25 \%$ difference between larger and smaller pieces in the rage. 
The elongation and flatness characteristics of the sand-sized particles (between $0.15 \mathrm{~mm}$ and $2.3 \mathrm{~mm}$ ) seems to be persistent across the investigated sand sizes, or, at least, a trend is not observed.

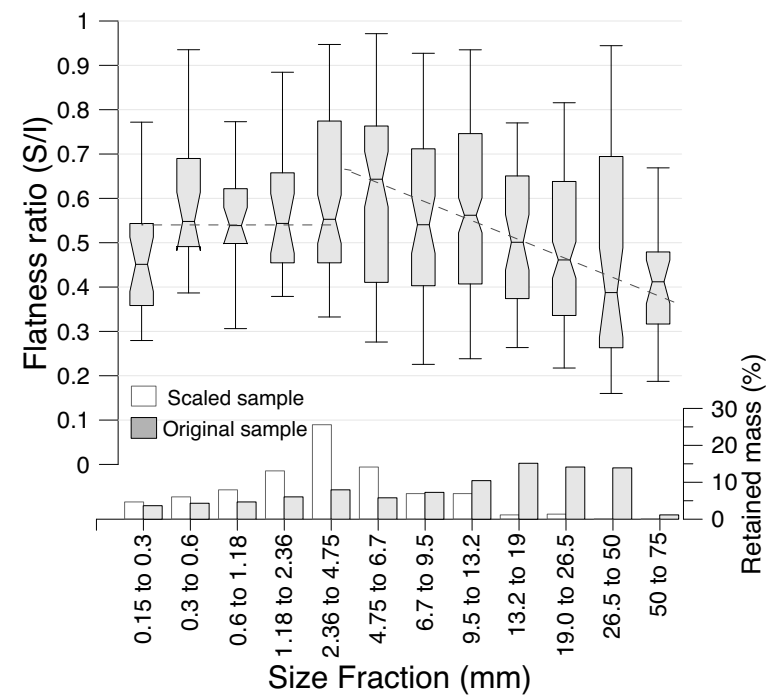

Fig. 7. Box plot showing the variability of flatness ratio, calculated from the form dimensions $S$ and $I$ for the different size fractions compared to the represented proportion of mass.

\subsection{Particle roundness}

Most of the particles classify as sub-rounded. A correlation with particle size or a particular trend cannot be identified from results.

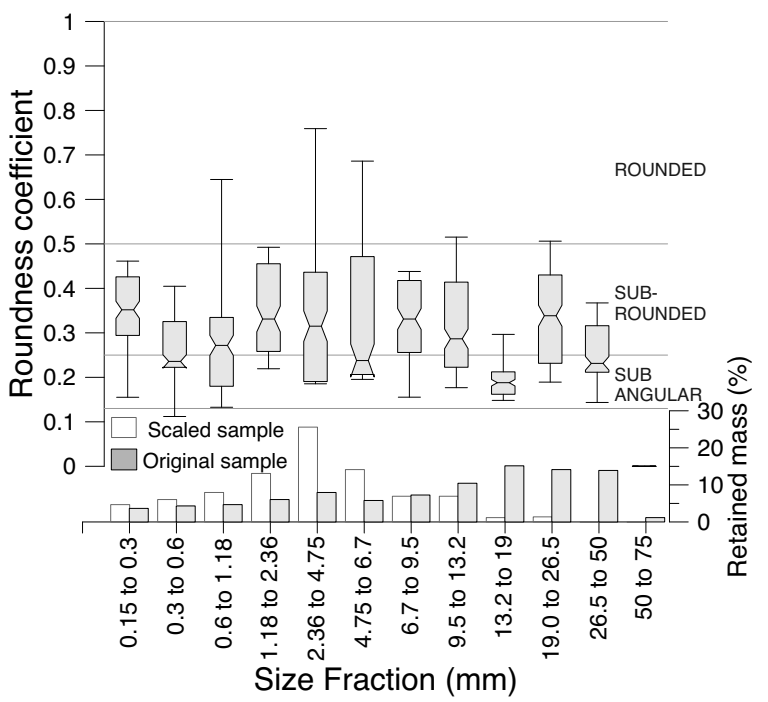

Fig. 8. Roundness data for the different size fractions compared to the represented proportion of mass.

\section{Discussion}

The elongation ratio of the particles composing the original and the scaled sample is not very different. Most of the material (by weight) both in the original and the scaled samples (sizes $9.5 \mathrm{~mm}$ to $50 \mathrm{~mm}$ and $1.18 \mathrm{~mm}$ to $6.7 \mathrm{~mm}$ respectively) contains particles that have relatively similar elongation ratios. However, the differences in flatness ratio are more significant with up to $20 \%$ difference in median values when comparing the dominant size fractions, by weight, for the original samples and the scaled sample.

In any case, the scaled sample is less polydisperse in shape and its particles tend to be more regular compared with the original sample.

\section{Conclusions}

For the colluvial material studied, the trends in shape across particle size scales are small but potentially significant. Larger particles of the analysed material tend to be more platy or discoidal (less elongated and flatter) than smaller particles. Scaling inevitably alters the characteristic shapes of the material particles because it involves removing platy particles and replacing them with blockier pieces. The magnitude of the alteration depends on the scaling factor. The larger the degree of scaling, the larger the changes to particle shape induced by scaling.

The questions remain: how much impact do the changes in shape due to scaling have on shear strength, or alternatively, how much change in shape can be tolerated before it has a significant impact on measured strength. Resolving these questions may require both physical testing and DEM numerical modelling.

The fabric of the particles characterised by lamination may explain the variability in flatness ratio, as there is a preferential tendency for the particles to break along laminations, which are characteristically oriented perpendicular to their thickness (minimum dimension). Therefore, the origin of the material is important to the appreciation of shape effects related to scaling.

No trend is observed with size in particle roundness across size ranges, so, roundness is unlikely to add a shape effect when scaling, in the particular case of the studied colluvial material.

Thanks to Fortescue Metals Group Ltd for providing the material and funding this research along with SRK Consulting and the University of Newcastle.

\section{References}

1. T. Leps, J Soil Mech Found Div, 96 1159-1170 (1970)

2. W. Holtz, H. Gibbs, J Soil Mech Found Div, 82 122 (1956)

3. D. Nguyen, E. Azema, P, Sornay, F. Radjai, Phys. Rev. E, 91032203 (2015)

4. Z. Lewy, IJG, 3 226-236 (2012)

5. C. Simpson, D. Storey, D. Kepert, R. Boyd, N. Nitschke, Iron Ore Conference (2011)

6. Krumbein, W.C. J. Sed. Petrol., 11, 64-72 (1941)

7. S. Blott, and K. Pye, Sedimentology, 55, 31-63 (2008).

8. Bagheri 2015, Powder Technology, 270 141-153 (2015)

9. H. Wadell, The Journal of Geology, 40, 443-451 (1932) 